\title{
The Clinical Application of Non-invasive Prenatal Genetic Testing in the Screening of Fetal Chromosomal Diseases
}

\section{Yu Pang}

Anhui Medical University Affiliated Maternity and Child Health Hospital: Anhui Province Maternity and Child Health Hospital

chaohong wang

Anhui Medical University Affiliated Maternity and Child Health Hospital: Anhui Province Maternity and Child Health Hospital

\section{Junxiang Tang}

Anhui Medical University Affiliated Maternity and Child Health Hospital: Anhui Province Maternity and Child Health Hospital

Jiansheng Zhu (D 593130772@qq.com )

Maternity and Child Health Hospital of Anhui Province, Hefei, China

\section{Research}

Keywords: Non-invasive prenatal genetic testing,Screening efficacy,Chromosome aneuploidy

Posted Date: April 6th, 2021

DOl: https://doi.org/10.21203/rs.3.rs-297925/v1

License: (c) (i) This work is licensed under a Creative Commons Attribution 4.0 International License. Read Full License 


\section{Abstract}

Objective $\mathbb{X}$ To explore the efficacy and clinical application value of non-invasive prenatal testing (noninvasive prenatal testing, NIPT) for screening fetal chromosomal abnormalities.

Methods: NIPT was performed on 25,517 pregnant women. The high-risk samples were compared with amniotic fluid and cord blood chromosome karyotype analysis. Some samples were further verified by microarray (CMA), and pregnancy outcomes were followed up.

Results: Of all the cases examined, 25502 cases were detected successfully, and a total of 294 high-risk samples $(1.15 \%)$ were detected, of which further diagnosis was made in 208 cases, true positive samples were detected in 96 cases, and further tests were refused in 86 cases. 71 cases $(0.28 \%)$ of autosomal aneuploid high-risk samples were detected and 51 cases were diagnosed, including 44 cases of trisomy 21 (T21), 5 cases of trisomy 18 (T18), and 2 cases of trisomy 13 (T13). The PPV was $90.90 \%, 45.45 \%$ and $33.33 \%$, respectively. Thirteen high-risk samples of trisomy 21,18 , and 13 were detected, and 1 case was confirmed as T21 mosaic PPV was $8.33 \%$ NPV was $100 \%$. High-risk samples of sex chromosome aneuploidy (SCA) were detected in 72 cases $(0.28 \%), 23$ cases were diagnosed, and the PPV was $40.07 \%$. The PPV was $12.00 \%, 50.00 \%, 72.73 \%$ and $75.00 \%$, respectively, and the PPV was $12.00 \%, 50.00 \%$, $72.73 \%$ and $75.00 \%$, respectively. High-risk samples of copy number variation (CNV) were detected in 104 cases $(0.41 \%)$, and 18 cases were diagnosed, with a PPV of $32.14 \%$. Other high-risk samples of chromosome aneuploidy were detected in 34 cases $(0.13 \%)$, and 3 cases were diagnosed as T2, T9, and T16, respectively. PPV is $8.70 \%$.

Conclusion: NIPT is suitable for trisomy 21,18 , and 13 screening, especially for $\mathrm{T} 21$. It also has a certain

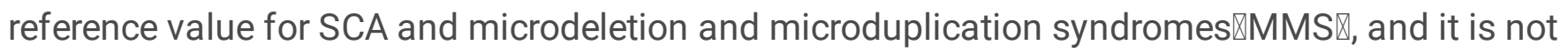
recommended for screening for other chromosomal aneuploidies.

\section{Background}

With the liberalization of my country's birth policy, the number of births in my country has gradually increased each year, and the total incidence of birth defects has also increased. The annual incidence of birth defects in my country is about $5.6 \%$, and the number of new cases is increasing annually by 900,000 . Among them, Down Syndrome (DS) adds 23,000 to 25,000 people every year. Chromosomal disease is one of the most common causes of birth defects. At present, prenatal screening is an effective method to prevent birth defects ${ }^{[1]}$. Traditional serological screening uses pregnant women's serum markers such as AFP, $\beta$-hCG, uE3, and inhibin-A to find high-risk pregnant women. However, its screening accuracy is poor and the missed diagnosis rate is high ${ }^{[2]}$. Although amniocentesis and chorionic sampling have high accuracy, they are not easy to be accepted and promoted because of their invasive operations, which can easily cause anxiety, fear, and risks of miscarriage and intrauterine infection in pregnant women $\left.{ }^{[3]}\right]$ 
In 1997, Professor Yuming Lu of the Chinese University of Hong Kong proved for the first time that there is fetal free DNA in the peripheral blood of pregnant women, opening up a new method of non-invasive prenatal testing ${ }^{[4]}$. So far, it has been widely used in many countries around the world ${ }^{[5]}$. The non-invasive prenatal test (NIPT) uses a new generation of high-throughput sequencing technology to detect free fetal DNA based on maternal plasma. After bioinformatics analysis, the fetal chromosomal abnormalities are detected, which is non-invasive, safe, and Fast detection, early detection time, high accuracy and reliability, and other technical advantages.NIPT not only has high sensitivity and specificity for autosomal aneuploidy diseases but also for sex chromosome aneuploidy, another chromosome aneuploidy, and chromosome submicroscopic structure (MMS) Screening has certain clinical practical value ${ }^{[6]}$. This study selected 25,517 pregnant women who underwent NIPT screening in our hospital from September 2019 to September 2020 to analyze the effectiveness of NIPT in screening fetal chromosomal abnormalities and explore its clinical application value.

\section{Object}

Selected from September 2019 to September 2020 in Anhui Province Maternity and Child Health Hospital for NIPT detection of 25,517 pregnant women, aged 16 to 48 years old, a gestational week from $11^{+5}$ to $30^{+6}$ Including singleton pregnancy and twin pregnancy. Inspection indications include: prenatal serological screening is high risk and borderline risk, that is, $T 21 \geq 1 / 270$ is a high risk, $T 18 \geq 1 / 350$ is a high risk, T21:1/270-1/1000, T18:1/350-1/1000, which is the critical risk; pregnant women with high age risk, missed screening, and pregnant women with voluntary NIPT without indication; The abnormalities suggested by ultrasound include cervical hyaline membrane thickness (nuchal translucency, NT) greater than $3 \mathrm{~mm}$, ventricular bright spots, abnormal echoes of the fetal choroid plexus, slightly widened lateral ventricles, and enhanced renal and bowel echoes. Excluding pregnant women with chromosomal diseases, in vitro fertilization (IVF), history of blood transfusion within 2 years, history of stem cell therapy, and transplantation may have an impact on the results of NIPT. All pregnant women have been informed of the necessity of prenatal diagnosis, the scope of adaptation of NIPT screening, and its limitations and related risks. All pregnant women in this study signed an informed consent form.

\section{Method}

\subsection{Specimen collection and non-invasive prenatal testing}

Take $10 \mathrm{ml}$ of pregnant women's peripheral blood for anticoagulation with EDTA, and transport it within 8 hours at $4^{\circ} \mathrm{C}$, or store it in a Streck tube $\left(6^{\circ} \mathrm{C} \varangle 35^{\circ} \mathrm{C}\right)$ and transport it within 96 hours. Or separate plasma at 1600rpm and 10min twice within 24 hours, extract fetal DNA, construct a library, use the ILLumination Hiseq500 sequencer to perform high-throughput sequencing, bioinformatics analysis, and compare human genome reference sequence maps, and use the non-invasive prenatal screening analysis software Illumina Sequencing Analysis Viewer 1.9.1 to calculate the risk rate and $Z$ value of chromosomes, $-3 \llbracket Z$

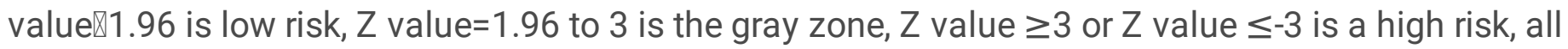


pregnant women with a high risk of NIPT results receive genetic counseling, and it is recommended An interventional prenatal diagnosis program was selected to verify the NIPT results and confirm the diagnosis.

\subsection{Karyotype analysis of amniotic fluid chromosome}

Amniotic fluid samples are collected for pregnant women with a high risk of NIPT, and an interventional prenatal diagnosis program is selected: under the guidance of ultrasound, amniocentesis is performed, $20 \mathrm{ml} 30 \mathrm{ml}$ of amniotic fluid is drawn, cultured in a constant temperature $\mathrm{CO}_{2}$ incubator at $37^{\circ} \mathrm{C}$, and the harvest is split During the period of the vigorous cell, some pregnant women undergo umbilical cord blood puncture sampling, cell culture, film production, and fetal chromosome karyotype analysis.

\subsection{Chromosome microarray analysis of amniotic fluid}

For pregnant women whose NIPT test result is CNV, choose amniotic fluid chromosome microarray analysis (CMA): amniotic fluid samples are centrifuged, precipitated, and genomic DNA is extracted. SNP array is performed according to the Illumina company chip operation steps, data is collected and the results are analyzed.

\subsection{Data analysis and follow-up of pregnancy outcome}

Analyze the NIPT test data, and calculate the number of true positive, false positive, true negative, false negative, positive predictive value (PPV), negative predictive value (NPV), sensitivity, and specificity. $\mathrm{PPV}=$ number of true positive cases screened/the number of actual positive cases $\times 100 \%, N P V=$ number of true negative cases screened/number of actual negative cases $\times 100 \%$, sensitivity=number of true positive cases screened/(true positive cases Number + number of false-negative cases) $\times 100 \%$, specificity $=$ number of true negative cases screened out / (number of true negative cases + number of false-positive cases) $\times 100 \%$, At the same time, conduct telephone follow-up of pregnancy outcome or check the registration form of follow-up of pregnant women and record whether there are miscarriage, labor induction, delivery, and false-negative samples.

\section{Result}

\subsection{Screening status}

A total of 25,517 samples were collected. The collected sample information includes the experimental sample number, storage temperature, blood draw date, age, weight, gestational week at the time of examination, date of last menstruation, expected date of delivery, number of pregnancy, presence or absence of Down's screening, and the presence or absence of type-b ultrasonic, single and twin 
pregnancies, IVF, pregnancy outcome, history of allogeneic blood product transfusion, history of stem cell treatment and transplantation, history of the chromosomal disease in the husband, telephone number and address of registration return visit, all samples were interviewed by telephone, and high-risk samples were interviewed by telephone several times. It is recommended to further intervene in prenatal diagnosis. No false-negative cases were found after the follow-up of samples with a low-risk NIPT result. There was no false-negative registration in the follow-up registration form during pregnancy.

Among the 25,517 samples, 52 cases needed to be re-collected due to hemolysis, the $Z$ value was in the gray zone twice, and the fetal DNA concentration was too low. 15 cases were still unable to meet the testing requirements after the re-sampling. refund processing according to test failure.25,502 samples were successfully tested, and a total of 294 high-risk samples of chromosomes were screened. Among them, 208 high-risk NIPT samples were selected for interventional prenatal diagnosis. 96 cases were found to be true positives, and 86 cases were rejected for further testing(Table 1).

Table 1

Two high-risk sample situations at the same time

\begin{tabular}{|llllll|}
\hline $\begin{array}{l}\text { Sample } \\
\text { number }\end{array}$ & Age & $\begin{array}{l}\text { Gestational } \\
\text { week }\end{array}$ & $\begin{array}{l}\text { NIPT } \\
\text { high risk } \\
1\end{array}$ & $\begin{array}{l}\text { NIPT } \\
\text { high risk 2 }\end{array}$ & $\begin{array}{l}\text { Diagnostic } \\
\text { result }\end{array}$ \\
\hline 20AH04289 & 26 & 18 & T13 & $47 X X X$ & $46, X N$ \\
\hline 20 AH00875 & 30 & $22^{+2}$ & T20 & $47, X X Y$ & $46, X N$ \\
\hline AB198R04200 & 39 & $17^{+4}$ & T18 & $\begin{array}{l}\text { Too many Sex } \\
\text { chromosomes }\end{array}$ & $46, \mathrm{XN}$ \\
\hline AB198R00291 & 34 & $21^{+2}$ & T21 & T13 & $46, \mathrm{XN}$ \\
\hline 20AH05459 & 31 & $18^{+1}$ & $45, \mathrm{XO}$ & T7 & $46, \mathrm{XN}$ \\
\hline
\end{tabular}

\subsection{NIPT's detection efficiency on T21, T18, T13}

For samples with a high risk of T21, T18, T13 detected by NIPT, karyotype analysis was performed, and some samples were tested with chromosome karyotype and gene chip technology, and T21, T18, T13 were all correctly detected. T21: 48 cases $(0.19 \%), 44$ true positives; T18: 15 cases $(0.06 \%), 5$ true positives; T13: 8 cases $(0.04 \%), 2$ true positives. The PPV of T21, T18, and T13 were $90.90 \%, 45.45 \%$, and $33.33 \%$. The specificities of NIPT for T21, T18, and T13 were $99.98 \%, 99.98 \%$, and $99.98 \%$. no false- 
negative cases of T21, T18, T13 have been recorded of the T18 and T13 samples without further karyotype verification, 4 cases ( 3 cases in T8, 1 case in T13) induced labor or spontaneous abortion in the local hospital due to abnormalities or abnormalities of the fetus detected by B-ultrasound, and 2 cases (T18, T13 each 1 case) went directly to the local hospital to induce labor and did not conduct genetic and pathological examinations on the induced labor or spontaneous abortion tissue. This is also the limitation of this study. NIPT screened confirmed cases of T21, T18, and T13, and all chose to terminate the pregnancy.

Table 2

The efficacy of NIPT in screening autosomal aneuploidy T21, T18, T13

\begin{tabular}{|cllllllllll|}
\hline NIPT & $\begin{array}{l}\text { Number } \\
\text { of } \\
\text { positive } \\
\text { cases }\end{array}$ & $\begin{array}{l}\text { Amniotic } \\
\text { fluid } \\
\text { puncture } \\
\text { cases }\end{array}$ & TP & FP & FN & TN & Sensitivity & Specificity & PPV & NPV \\
T21 & 48 & 48 & 44 & 4 & 0 & 25454 & 100 & 99.98 & 90.90 & 100 \\
T18 & 15 & 11 & 5 & 6 & 0 & 25491 & 100 & 99.98 & 45.45 & 100 \\
\hline T13 & 8 & 6 & 2 & 4 & 0 & 25496 & 100 & 99.98 & 33.33 & 100 \\
\hline
\end{tabular}

\subsection{NIPT's detection efficiency for SCA and CNV}

For the SCA and CNV samples that NIPT indicated as high risk, karyotype analysis was performed, and some samples were tested by karyotype and gene chip. A total of 72 cases $(0.28 \%)$ were detected for SCA and 104 cases $(0.41 \%)$ for CNV. No false-negative cases have been recorded. The test results are as follows: Among 72 cases of SCA, 23 were true positives, 33 were false positives, and PPV was $40.07 \%$. Among them, 45, X0, 33 cases, 3 true positives, 22 false positives, PPV 12.00\%; 47, XXY, 14 cases, 6 true positives, 6 false positives, PPV 50.00\%; 47, XXX, 17 cases.Among the cases, 8 were true positives, 3 were false positives, PPV 72.73\%; 47, XYY, 8 cases, 6 were true positives, 2 were false positives, PPV $75.00 \%$. The detection efficiency is $47 X Y Y>47 X X X>47 X X Y>45 X 0$. Among the 23 pregnancy outcomes with SCA true positive samples, 17 cases were induced to labor, 5 cases were born, and the current phenotypes are all normal, and 1 case continues to be pregnant. Among 104 CNV cases, 18 were true positives and 38 were false positives, with a PPV of $32.14 \%$ (Table 3).

Table 3

The efficacy of NIPT in screening SCA and CNV 


\begin{tabular}{|lllllllll|}
\hline NIPT & $\begin{array}{l}\text { Number of } \\
\text { positive cases }\end{array}$ & $\begin{array}{l}\text { Amniotic fluid } \\
\text { puncture } \\
\text { cases }\end{array}$ & TP & FP & FN & TN & Specificity & PPV \\
\hline SCA & 72 & 56 & 23 & 33 & 0 & 25446 & 99.87 & 40.07 \\
\hline $45 X 0$ & 33 & 25 & 3 & 22 & 0 & 25477 & 99.91 & 12.00 \\
\hline $47 X X Y$ & 14 & 12 & 6 & 6 & 0 & 25490 & 99.97 & 50.00 \\
\hline $47 X X X$ & 17 & 11 & 8 & 3 & 0 & 25491 & 99.99 & 72.73 \\
\hline $47 X Y Y$ & 8 & 8 & 6 & 2 & 0 & 25484 & 99.99 & 75.00 \\
\hline CNV & 104 & 56 & 18 & 38 & 0 & 25446 & 99.85 & 32.14 \\
\hline
\end{tabular}

\subsection{NIPT's detection efficiency for other chromosomes}

Based on NIPT for whole-genome sequencing, we also analyzed the detection results of other chromosomal abnormalities in NIPT. A total of 34 cases $(0.13 \%)$ of other chromosomal abnormalities were detected, and the PPV was $8.70 \%$. Among them, 3 cases were true positive: 1 case each for T2, T9, and T16, and their PPV was $50.00 \%, 25.00 \%$, and $25.00 \%$, respectively. There were 21 false positives and 10 unverified cases. Among the false-positive samples, T7 was the most detected, followed by T8, T10, and T16, and the rest were very few. Pregnancy outcome: T2 sample, the child has been born, the phenotype is normal. T9 sample, choose to induce labor. T16 sample, continue the pregnancy. Besides, 13 high-risk samples of trisomy 21,18 , and 13 were detected, and the total PPV was $8.33 \%$. One true positive case (sample number 20AH00515) was T21mos. The child was born with a normal phenotype. One case was not verified (sample number 19AH03199, direct labor induction), and the rest were false positives(Table 4).

\section{Table 4}

The efficacy of NIPT in screening other chromosomal aneuploidies 


\begin{tabular}{|llllll|}
\hline NIPT & Positive & TP & FP & Unverified & PPV \\
\hline T1 & 1 & 0 & 1 & 0 & 0 \\
\hline T2 & 2 & 1 & 1 & 0 & 50.00 \\
T3 & 1 & 0 & 1 & 0 & 0 \\
\hline T7 & 9 & 0 & 3 & 6 & 0 \\
\hline T8 & 5 & 0 & 3 & 2 & 0 \\
\hline T9 & 2 & 1 & 1 & 0 & 50.00 \\
\hline T10 & 3 & 0 & 3 & 0 & 0 \\
\hline T12 & 1 & 0 & 1 & 0 & 0 \\
\hline T14 & 1 & 0 & 1 & 0 & 0 \\
\hline T15 & 2 & 0 & 2 & 0 & 0 \\
\hline T16 & 4 & 1 & 3 & 0 & 25.00 \\
\hline T17 & 1 & 0 & 0 & 1 & 0 \\
\hline T20 & 2 & 0 & 1 & 1 & 0 \\
\hline T21mos & 5 & 1 & 3 & 1 & 25.00 \\
\hline T18mos & 5 & 0 & 5 & 0 & 0 \\
\hline T13mos & 3 & 0 & 3 & 0 & 0 \\
\hline
\end{tabular}

\subsection{NIPT prompts CNV and amniotic fluid karyotype and CMA test results}

Among the 56 cases of CNV interventional diagnosis samples, 18 were true positive samples, 11 were micro-duplicate samples, 7 were micro-deletion samples, and 5 were pathogenic. There were 3 cases of possible pathogenicity, 10 cases of unclear clinical significance, and 1 case of abnormal karyotype. Induced labor in 4 cases; gave birth at full-term, 13 cases of children with normal phenotype; 1 case of continued pregnancy. CNV confirmed by chromosome karyotype and gene chip to confirm the true positive samples and pregnancy outcome囚Table 5 Figure 1 囚.

\section{Discussion}

Chromosome disease is one of the most serious birth defects in newborns. At present, there is no effective treatment, prenatal screening is a more effective way to reduce birth defects. NIPT is an 
important means of prenatal screening. In 2012, (ISPD) of the International Institute of Prenatal diagnosis and (ACOG) of the American College of Obstetrics and Gynaecology also recommended NIPT as a detection method for high-risk groups of chromosome aneuploidy ${ }^{[788]}$. Through the screening of NIPT in 25502 pregnant women, the screening efficiency of NIPT in fetal autosomal, sex chromosome, and other chromosome aneuploidy and CNV was analyzed, and its clinical value was explored. In this study, NIPT suggested that there were 48 cases $(0.19 \%)$ of high-risk T21, 15 cases $(0.06 \%)$ of T18, and 8 cases ( $0.04 \%$ ) of $\mathrm{T} 13$. The PPV of $\mathrm{T} 21, \mathrm{~T} 18$, and $\mathrm{T} 13$ is $90.90 \%, 45.45 \%$, and $33.33 \%$, respectively. It is consistent with the literature report ${ }^{[9]}$. The decrease of PPV, in turn, depends not only on the sensitivity of NIPT but also on the decrease of incidence of T21, T18, and T13. At the same time, due to the absence of chromosome karyotype examination in the tissues of induced labor or spontaneous abortion, it may contain some true positive samples of NIPT, which also affects PPV. This study shows that NIPT has high accuracy and reliability in screening $\mathrm{T} 21, \mathrm{~T} 18$, and $\mathrm{T} 13$, especially for $\mathrm{T} 21^{[10]}$.

Besides, 13 cases of T21mos, T18mos, and T13mos were detected in this study, and 1 case of true positive (T21mos) sample number was $20 \mathrm{AH} 00515,30$ years old, 20 weeks pregnant for 4 weeks. The results of Down's screening were as follows: T21ه1/620, chromosome karyotype indicated that $21 \mathrm{q} 21.1$ repetition was $1.76 \mathrm{Mb}$, involving one OMIM gene, and there was no report of repetitive pathogenicity of CLINGEN. The clinical significance of the repetition was unknown. After genetic counseling, we chose to continue pregnancy and now delivered, and the baby was born as a normal girl. The detection efficiency of NIPT for trisomy 21, 18, and 13 was significantly lower than that of NIPT for T21, T18, and T13. The analysis of the reason may be due to the non-separation of mitosis in the early embryo or the delayed division in the later stage, in which the multicellular system is composed of normal karyotype and aberrant karyotype makes it difficult to detect NIPT. The free DNA detected by NIPT comes from placental trophoblast cells, not a fetus. The inconsistency of chimerism in different regions of placental tissue leads to more false-positive NIPT detection ${ }^{[11]}$, especially in distinguishing true chimerism from false chimerism or low proportion chimerism. Therefore, NIPT is not recommended for screening chimeric chromosomal abnormalities.

In 72 cases of a sex chromosome aneuploidy, the detection rate of SCA was $0.28 \%$. The detection rate of PPV for sex chromosome aneuploidy was $40.07 \%$. Among them, 3, 6, 8 and 6 cases were true positive cases for $45 \llbracket X 0,47 \rrbracket X X Y, 47 \llbracket X X X, 47 \llbracket X Y Y$, respectively. The detection efficiency of NIPT for sex chromosome aneuploidy was $12.00 \%, 50.00 \%, 72.73 \%$, and $75.0 \%$, respectively, which was not as effective as that for autosomal aneuploidy, and its efficiency for autosomal aneuploidy was lower than that for autosomal aneuploidy. The detection efficiency was the lowest (PPV 12.00\%) for $45 \llbracket X 0$, but moderate for 47区XXY, 47XXX, and 47区XYY (PPV 64.52\%), which was consistent with that reported in the literature ${ }^{[12]}$. Because $X$ and $Y$ chromosomes are highly homologous, it is not easy to detect, and many fragments of the $Y$ chromosome are similar to other chromosomes, so the accuracy of sequencing is reduced. Factors such as the deviation of cytosine and thymine content on the X chromosome, random inactivation of the $X$ chromosome, restricted placental chimerism (CPM), and maternal SCA can also reduce the specificity of NIPT and detection ${ }^{[13]}$. Although the equipment, $Z$ value, and concentration 
settings used in different experiments are different, which may have a certain impact on the detection results, NIPT still has certain clinical applicability for the detection of sex chromosome triploid, while the detection efficiency for $45 \rrbracket X O$ is not stable enough. In some cases of SCA, there is no obvious clinical phenotype and ultrasound abnormality in the fetus. Phenotypic abnormality can not be found in the fetal and infantile follow-up. After puberty, it will have a serious impact on physiology and psychology. Therefore, prenatal screening, genetic counseling, and long-term follow-up of SCA are particularly important $^{[14]}$.

This study shows that the efficiency of NIPT in the detection of aneuploidy of other chromosomes is low and has obvious limitations. NIPT revealed chromosome abnormalities in 34 cases, true positive in 3 cases, false positive in 21 cases, and no further verification in 10 cases. T7 was the most detected, followed by $T 8, T 10$, and $T 16$, which was similar to that reported in the literature ${ }^{[15]}$. Its PPV is $12.50 \%$, which is significantly lower than autosomal aneuploidy and sex chromosome aneuploidy. The reason for the higher false positive may be that the incidence of trisomy of these rare chromosomes is very low. and related factors such as "trisomy self-rescue" in the process of embryogenesis, placental chimerism caused by mitotic errors of diploid embryos, maternal cell contamination, maternal tumor, and so on ${ }^{[160}$ 17]. The true positives of the 3 cases were T2, T9, and T16 respectively. T2 was the sample number of AB198R51420,43 years old, 20-5 weeks of gestation. The karyotype of amniotic fluid was $46 \mathrm{XN}$ chromosome 2 trisomy $30 \%$ verified by CMA. Fetal ultrasound examination was normal. According to the database query, some abnormal phenotypes were reported, such as oligohydramnios, abnormal face, congenital heart abnormalities, etc. and there were also reports that some fetuses had no obvious abnormal pregnancy outcome. After genetic counseling, we chose to continue the pregnancy, and the phenotype of the fetus was normal after birth. The sample number of T16 was AB19HG04493,33 years old, and the gestation was 15 to 5 weeks. The result of CNV-Seq verification was sep[hg19] dup (16) (p13.3q24.3) repetitive $90.35 \mathrm{Mb}$. There was no abnormality in B-ultrasound. After database inquiry, trisomy 16 showed fetal intrauterine limitation and a congenital heart defect. After genetic counseling, we chose to continue the pregnancy \at present, the fetus has been born.T9 is the sample number AB19HG06905,28 years old, 16 weeks pregnant and 4 weeks pregnant. Verified by the karyotype of amniotic fluid cells $\triangle \mathrm{CMA}:(46 \otimes X X[31] / 47 \otimes X X[59]) ; C M A$ results are $60 \otimes 70 \%$ trisomy of chromosome 9 . After database query: its phenotypic growth retardation, cognitive impairment, microphthalmia, heart abnormality, skeletal development abnormality, etc., may cause disease after genetic counseling, and choose to directly induce labor. Therefore, NIPT should combine the routine examination of pregnant women and fetal B-ultrasound screening for other chromosome aneuploidy results, and provide information helpful to the judgment of results, genetic counseling, and clinical decision-making as far as possible, to reduce maternal anxiety and unnecessary pregnancy termination. Liang et al ${ }^{[18]}$ reported nearly 100000 samples and found that the detection rate of aneuploidy of other chromosomes was relatively low by expansive NIPT screening, with a PPV of $28 \%$. The authors believe that it may also be related to the low total incidence of chromosomal abnormalities mentioned above. CPM. (ACMG) of the American Society of Medical Genetics and Genomics pointed out in 2016 that NIPT is not suitable for the detection of autosomal aneuploidy other than $\mathrm{T} 21, \mathrm{~T} 18$, and $\mathrm{T} 13^{[19]}$. Therefore, NIPT as a fetal aneuploid 
detection of other chromosomes, the accuracy is insufficient, the use of NIPT to detect other chromosome aneuploidy should fully inform pregnant women of its limitations, the results need more verification.

In the detection of chromosome MMS by NIPT, CNV samples were detected in 104 cases, 18 cases were true positive, PPV was $32.14 \%$, and 38 cases were false positive. NIPT has a certain detection efficiency for CNV, but there are still many false positives, and the results still need to be further verified by chromosome karyotype analysis or CMA detection [20ख21], which is especially important for pathogenic or possibly pathogenic CNV. Although there are many false positives of MMS detected by NIPT, it is difficult to find that MMS, NIPT below $10 \mathrm{Mb}$ can make up for the deficiency of chromosome karyotype analysis and reduce the missed diagnosis caused by artificial visual judgment. The results of this study suggest that the conventional sequencing depth of NIPT is less effective for CNV detection, and the reason may be related to the refusal of further CMA verification in some samples, and the interference of low fetal free DNA level restricted placental chimerism and abnormal maternal chromosome copy number ${ }^{[22 \times 23]}$, which may also be the common reason for the low efficiency of multiple high-risk samples (Table 1). Therefore, when the fetus is CNV detected by NIPT, we should further understand the medical history of pregnant women, combined with the results of prenatal ultrasound diagnosis, consider the need for interventional prenatal diagnosis, and combine chromosome karyotype analysis and CMA detection techniques to further improve the detection rate of abnormal chromosomes ${ }^{[24 \times 25826]}$, and select fetal parents' chromosomes for control analysis if necessary. Among the true positive samples of CNV in this study (Table 5), there were 5 cases of pathogenicity, 3 cases of possible pathogenicity, and 10 cases of unknown clinical significance. Sample 19AH05194:NIPT reported that there were $2.5 \mathrm{Mb}$ duplicates in the $3 p 26.3$ region, and the chip results showed that $\operatorname{arr}[\mathrm{HG} 19] 3 \mathrm{p} 26.3(285856-2499708) \times 3$ involved three OMIM genes, including CHL (607416), CNYN (607220), and CNTN (607280). Some patients with autism, cognitive impairment, and epilepsy were found in PUBMED to repeat with this area, and the repetition was possible to cause disease. After genetic counseling, choose to continue the pregnancy, the fetus has been born, normal growth and development, parents refused to do carrier testing. Sample 20AH01147: the chip result showed that the $2 q 13$ deletion was $2.12 \mathrm{Mb}$, which was possible pathogenicity, which was consistent with its mother's CMA test sample (serial number CMA20200277). It was caused by maternal inheritance and chose to continue the pregnancy. the fetus has been born and its growth and development are normal. Sample 20AH00928: no karyotype was performed. B-ultrasound showed that fetal left hydronephrosis with left ureter dilatation, permanent left superior vena cava, and widened coronary sinus. Chip results: there were multiple abnormalities on chromosome 8, 8p23.1p12 region deletion involved 8p23.1 deletion syndrome, and the characteristic phenotypes were congenital heart abnormality, renal abnormality, growth retardation, and mental retardation. 8p12q24.3 region repetition involves trisomy 8 , which is characterized by mental retardation, a special face, heart abnormality, and so on. After genetic counseling for pathogenic CNV, pregnant women chose to induce labor. Sample 20AH02846: the karyotype of amniotic fluid was $46 \mathrm{del}(5)(\mathrm{p} 14)$. The result of microarray showed that there was a 5p15.33-p14.3 deletion of $22.5 \mathrm{Mb}$, involving 56 OMIM genes. The deletion in this region involved Cri-du-chat syndrome. The main phenotypes included mental retardation, language disorder, catlike crying in infancy, and so on. The deletion was not abnormal in peripheral blood karyotype of 
pathogenic CNV, parents, and induced labor after genetic counseling. The sample AB198R02189: chip result is $1 \mathrm{q} 21.1 \mathrm{q} 21.2 .71 \mathrm{MB}$, involving $13 \mathrm{OMIM}$ genes. The repetition in this region is related to $1 \mathrm{q} 21.1$ microrepetition syndrome, and the main phenotypes are mild to moderate mental retardation, autism, hyperactivity disorder, tycoon, etc $邓$ the syndrome is not complete, and the penetrance rate is $29.1 \%$. The repetition is pathogenic $\mathrm{CNV}$, after genetic counseling, choose to terminate pregnancy. Therefore, from the above sample analysis, it can be seen that the detection of CNV with clear pathogenicity by NIPT is of clinical significance and can provide an important basis for subsequent karyotype analysis or CMA detection ${ }^{[27]}$. In recent years, scalable NIPT checking has helped to further improve the performance of MMS detection, but the 2015 ISPD update guidelines still limit NIPT to the scope of MMS that has been studied $^{[28]}$.

In summary, as an important method of prenatal screening, NIPT has high accuracy in the screening of trisomy 21,18 , and 13 with a higher incidence, especially trisomy 21 , which is very effective for SCA. And MMS testing also has a certain reference value. But it is not recommended for the detection of other chromosomal aneuploidies. We believe that in the future, by upgrading the sequencing technology process, improving the bioinformatics analysis algorithm and library construction, high-precision collection of free fetal DNA, and increasing the coverage of the whole genome,it may further improve the detection efficiency of NIPT, combined with maternal medical history examination, serology, and Ultraacoustic auxiliary inspection, chromosome karyotype analysis, fluorescence in situ hybridization technology, CMA detection technology, etc. can better utilize the advantages of NIPT technology.

\section{Declarations}

\section{Ethics approval and consent to participate}

The study was approved by the Hospital Ethics Committee of Anhui Maternity and Child Health Care Hospital. Each patient signed informed consent prior to study enrollment.

\section{Consent for publication}

Not applicable.

\section{Availability of data and materials}

The data supporting the conclusions of this article is included within the article.

\section{Funding}

No Funding 


\section{Authors' contributions}

All authors have participated in the study and manuscript preparation.

\section{Acknowledgments}

We would like to thank our patients for agreeing to donate their personal data to our study and have these been published.

\section{References}

1. Krstić N, Običan SG. Current landscape of prenatal genetic screening and testing[J]. Birth Defects Res. 2020 ,1;112(4):321-331. doi: 10.1002/bdr2.1598. Epub 2019 Oct 21. PMID: 31633301.

2. Yin $L$, Tang $Y$, Lu Q,et al.Application value of NIPT for uncommon fetal chromosomal abnormalities[J]. Mol Cytogenet. 2020 Aug 28;13:39. doi: 10.1186/s13039-020-00508-z. PMID: 32874204; PMCID: PMC7456042.

3. Salomon LJ, Sotiriadis A, Wulff CB, Odibo A, Akolekar R. Risk of miscarriage following amniocentesis or chorionic villus sampling: systematic review of literature and updated meta-analysis[J]. Ultrasound Obstet Gynecol. 2019 Oct;54(4):442-451. doi: 10.1002/uog.20353. Epub 2019 Sep 6. PMID: 31124209.

4. Lo YM, Corbetta N, Chamberlain PF,et al. Presence of fetal DNA in maternal plasma and serum[J]. Lancet. 1997囚16;350(9076):485-487. doi: 10.1016/S0140-6736(97)02174-0. PMID: 9274585.

5. Gadsbøll K, Petersen OB, Gatinois V, et al. NIPT-map Study Group, Vogel I. Current use of noninvasive prenatal testing in Europe, Australia and the USA: A graphical presentation[J]. Acta Obstet Gynecol Scand. 2020区 99(6):722-730. doi: 10.1111/aogs.13841. Epub 2020 Apr 3. PMID: 32176318.

6. Samura Osamu,Update on noninvasive prenatal testing: A review based on current worldwide research.[J] .J Obstet Gynaecol Res, 2020, 46: 1246-1254.

7. Benn P, Borrell A, Crossley J, et al. International Society for Prenatal Diagnosis. Aneuploidy screening: a position statement from a committee on behalf of the Board of the International Society for Prenatal Diagnosis, January 2011[J].Prenat Diagn. 2011 『31(6):519-522. doi: 10.1002/pd.2730. PMID: 21604286.

8. American College of Obstetricians and Gynecologists Committee on Genetics. Committee Opinion No. 545: Noninvasive prenatal testing for fetal aneuploidy[J]. Obstet Gynecol. 2012 ه120(6):15321534. doi: 10.1097/01.AOG.0000423819.85283.f4. PMID: 23168792.

9. Norton ME, Jacobsson B, Swamy GK, et al.Cell-free DNA analysis for noninvasive examination of trisomy[J]. N Engl J Med. 2015 Apr 23;372(17):1589-1597. doi: 10.1056/NEJMoa1407349. Epub 2015 Apr 1. PMID: 25830. 
10. Mesoraca A, Margiotti K, Dello Russo C et al. Cell-free DNA screening for aneuploidies in 7113 pregnancies: single Italian centre study[J]. Genet Res (Camb). 2020 Jun 16;102:e5. doi: 10.1017/S001667232000004X. PMID: 32539871; PMCID: PMC7303798.

11. Li X, Ju D, Shi Y, et al.Fetal aneuploidy screening by non-invasive prenatal testing of maternal plasma DNA sequencing with "false negative" result due to confined placental mosaicism: A case report[J]. Medicine (Baltimore). 2020 Jul 17;99(29):e20848. doi: 10.1097/MD.0000000000020848. PMID: 32702826; PMCID: PMC7373535.

12. Zheng Y, Wan S, Dang Y, et al. Clinical experience regarding the accuracy of NIPT in the detection of sex chromosome abnormality[J]. J Gene Med. 2020 Aug;22(8):e3199. doi: 10.1002/jgm.3199. Epub 2020 May 28. PMID: 32267591.

13. McNamara CJ, Limone LA, Westover T, et al. Maternal source of false-positive fetal sex chromosome aneuploidy in noninvasive prenatal testing[J]. Obstet Gynecol. 2015 Feb;125(2):390-392. doi: 10.1097/AOG.0000000000000547. PMID: 25568987.

14. Yang Jiexia,Hou Yaping,Guo Fangfang et al. Noninvasive prenatal detection of fetal sex chromosome abnormalities using the semiconductor sequencing platform (SSP) in Southern China. [J] .J Assist Reprod Genet, 2021, Mar;38(3):727-734. doi: 10.1007/s10815-020-02056-2. Epub 2021 Feb 10. PMID: 33564935.

15. Scott F, Bonifacio M, Sandow R,et al. Rare autosomal trisomies: Important and not so rare[J]. Prenat Diagn. 2018 Sep;38(10):765-771. doi: 10.1002/pd.5325. Epub 2018 Jul 17. PMID: 29956348.

16. Liu Y, Liu H, He Y, et al.Clinical performance of non-invasive prenatal served as a first-tier screening test for trisomy $21,18,13$ and sex chromosome aneuploidy in a pilot city in China[J]. Hum Genomics. 2020 Jun 5;14(1):21. doi: 10.1186/s40246-020-00268-2. PMID: 32503639; PMCID: PMC7275506.

17. Luo $\mathrm{Y}, \mathrm{Hu} \mathrm{H}$, Jiang L,et al. A retrospective analysis the clinic data and follow-up of non-invasive prenatal test in detection of fetal chromosomal aneuploidy in more than 40,000 cases in a single prenatal diagnosis center[J]. Eur J Med Genet. 2020 Sep;63(9):104001. doi:

10.1016/j.ejmg.2020.104001. Epub 2020 Jul 2. PMID: 32622960.

18. Liang D, Cram DS, Tan H,et al.Clinical utility of non invasiveprenatal screening for expanded chromosome disease synd romes[J]. Genet Med. 2019 Sep;21(9):1998-2006. doi: 10.1038/s41436019-0467-4. Epub 2019 Mar 4.

19. Gregg AR, Skotko BG, Benkendorf JL, et al.Noninvasive prenatal screening for fetal aneuploidy, 2016 update: a position statement of the American College of Medical Genetics and Genomics[J]. Genet Med. 2016 Oct;18(10):1056-1065. doi: 10.1038/gim.2016.97. Epub 2016 Jul 28. PMID: 27467454.

20. Pei Y, Hu L, Liu J, et al. Efficiency of noninvasive prenatal testing for the daetection of fetal microdeletions and microduplications in autosomal chromosomes[J]. Mol Genet Genomic Med. 2020 Aug;8(8):e1339. doi: 10.1002/mgg3.1339. Epub 2020 Jun 15. PMID: 32543126; PMCID: PMC7434727.

21. Chen Y, Yu Q, Mao X,et al. Noninvasive prenatal testing for chromosome aneuploidies and subchromosomal microdeletions/microduplications in a cohort of 42,910 single pregnancies with 
different clinical features[J]. Hum Genomics. 2019 Nov 29;13(1):60. doi: 10.1186/s40246-019-02502. PMID: 31783780; PMCID: PMC6884830.

22. Wu X, Li Y, Xie X, et al.Clinical Review of Noninvasive Prenatal Testing: Experience from 551 Pregnancies with Noninvasive Prenatal Testing-Positive Results in a Tertiary Referral Center[J]. J Mol Diagn. 2020 Dec;22(12):1469-1475. doi: 10.1016/j.jmoldx.2020.09.008. Epub 2020 Oct 15. PMID: 33069877.

23. Hartwig TS, Ambye L, Sørensen S,et al. Discordant non-invasive prenatal testing (NIPT) - a systematic review[J]. Prenat Diagn. 2017 Jun;37(6):527-539. doi: 10.1002/pd.5049. Epub 2017 Jun 1. PMID: 28382695.

24. Xiang J, Ding Y, Song X,et al. Clinical Utility of SNP Array Analysis in Prenatal Diagnosis: A Cohort Study of 5000 Pregnancies[J]. Front Genet. 2020 Nov 6;11:571219. doi: 10.3389/fgene.2020.571219. PMID: 33240322; PMCID: PMC7677511.

25. Jiang N, Zhang Y, Song L,et al. [Application of high-throughput sequencing technology for the detection of fetal copy number variations][J]. Zhonghua Yi Xue Yi Chuan Xue Za Zhi. $2020 \mathrm{Jul}$ 10;37(7):779-784. Chinese. doi: 10.3760/cma.j.issn.1003-9406.2020.07.019. PMID: 32619264.

26. Caldwell S, Sagaser K, Nelson Z,et al. Deletion rescue resulting in segmental homozygosity: A mechanism underlying discordant NIPT results[J]. Am J Med Genet A. 2020 Nov;182(11):2666-2670. doi: 10.1002/ajmg.a.61801. Epub 2020 Aug 15. PMID: 32798301.

27. Yin Lianli,Tang Yinghua,Lu Qing et al. Application value of NIPT for uncommon fetal chromosomal abnormalities.[J] .Mol Cytogenet, 2020, Aug 28;13:39. doi: 10.1186/s13039-020-00508-z. PMID: 32874204; PMCID: PMC7456042.

28. Benn P, Borrell A, Chiu RW, et al.Position statement from the Chromosome Abnormality Screening Committee on behalf of the Board of the International Society for Prenatal Diagnosis[J]. Prenat Diagn. 2015 Aug;35(8):725-734. doi: 10.1002/pd.4608. Epub 2015 Jun 4. PMID: 25970088.

\section{Table}

Due to technical limitations, table 5 is only available as a download in the Supplemental Files section.

\section{Figures}




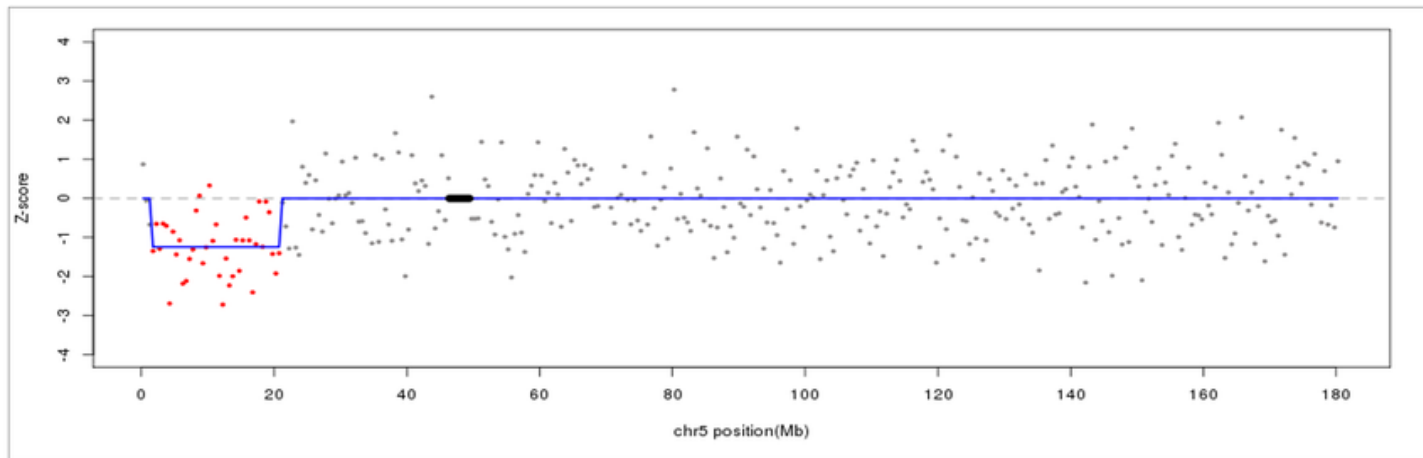

loss(chr5)(5p 15.33-p14.3)(1500000-20999999)(19500)(-1.24)

Karyotype analysis of amniotic fluid

$46, \mathrm{XX}, \operatorname{del}(5)(\mathrm{p} 14)$, The arrow shows the deletion of chromosome 5
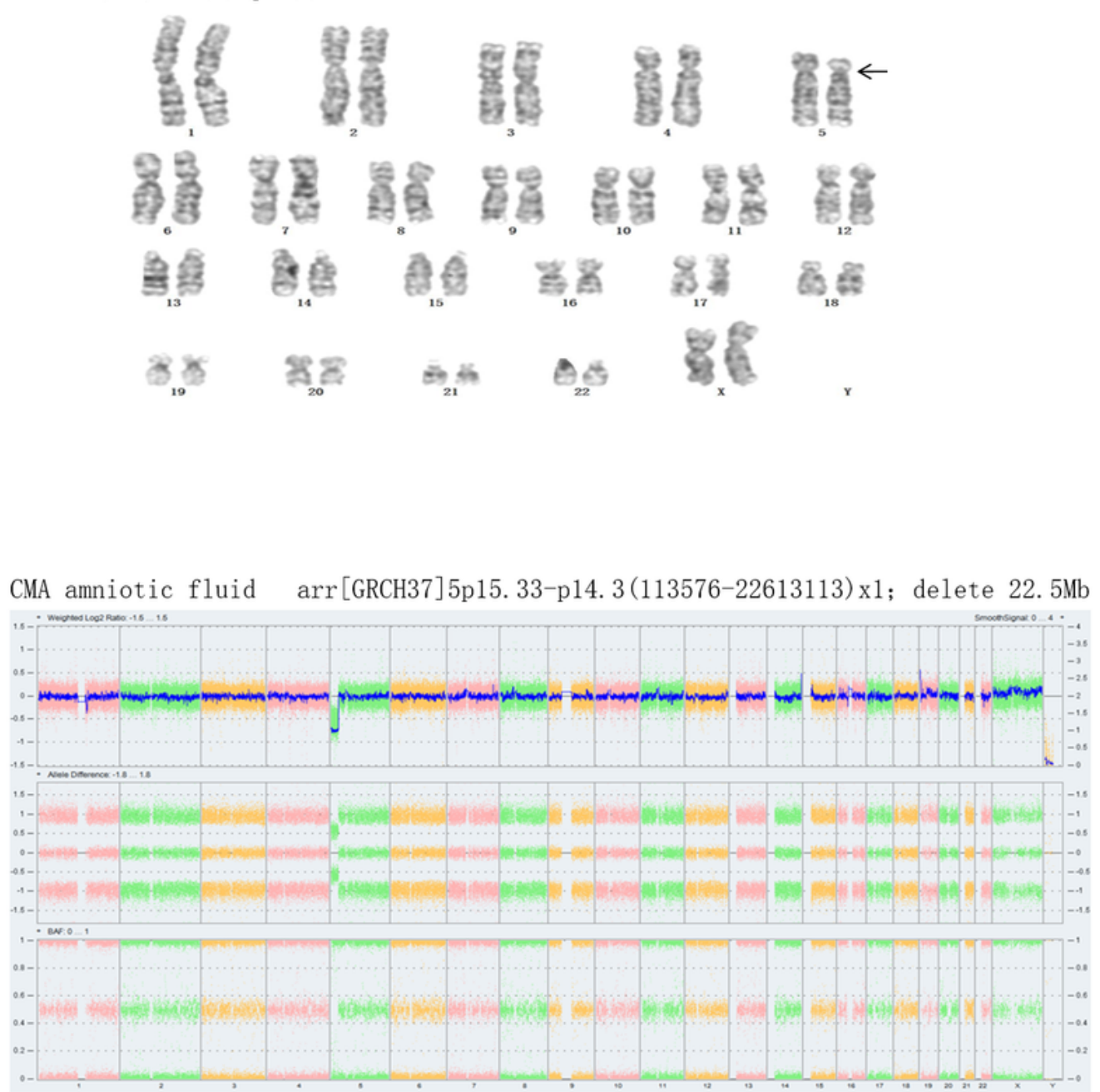

Figure 1

Comparison of chromosome karyotype and CMA in amniotic fluid of NIPT, with sample number 20AH02846 (diagnosed as Cri du chat syndrome) NIPT $₫ 5$ p15.33-p14.3 delete 19.5Mb囚

\section{Supplementary Files}


This is a list of supplementary files associated with this preprint. Click to download.

- Table5.docx 